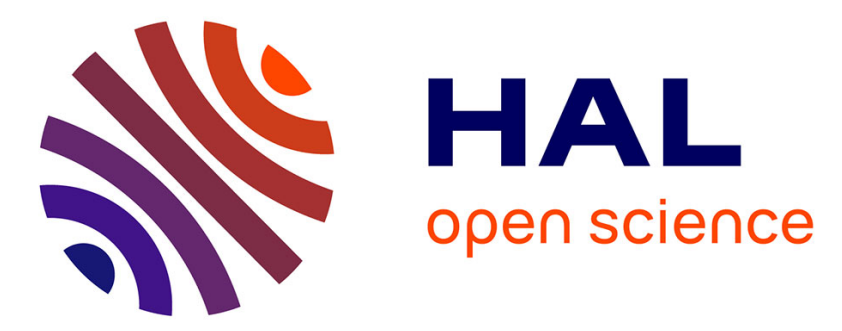

\title{
Ripple effect modelling of supplier disruption: integrated Markov chain and dynamic Bayesian network approach
}

Seyedmohsen Hosseini, Dmitry Ivanov, Alexandre Dolgui

\section{To cite this version:}

Seyedmohsen Hosseini, Dmitry Ivanov, Alexandre Dolgui. Ripple effect modelling of supplier disruption: integrated Markov chain and dynamic Bayesian network approach. International Journal of Production Research, 2020, 58 (11), pp.3284-3303. 10.1080/00207543.2019.1661538 . hal-02923276

\section{HAL Id: hal-02923276 \\ https://hal.science/hal-02923276}

Submitted on 26 Aug 2020

HAL is a multi-disciplinary open access archive for the deposit and dissemination of scientific research documents, whether they are published or not. The documents may come from teaching and research institutions in France or abroad, or from public or private research centers.
L'archive ouverte pluridisciplinaire HAL, est destinée au dépôt et à la diffusion de documents scientifiques de niveau recherche, publiés ou non, émanant des établissements d'enseignement et de recherche français ou étrangers, des laboratoires publics ou privés. 


\title{
Ripple Effect Modeling of Supplier Disruption: Integrated Markov Chain and Dynamic Bayesian Network Approach
}

\author{
Seyedmohsen Hosseini \\ Industrial Engineering Technology, University of Southern Mississippi, Long Beach, Mississippi, USA \\ Dmitry Ivanov* \\ Berlin School of Economics and Law, Berlin, Germany \\ Alexandre Dolgui \\ Automation, Production and Computer Sciences Department, IMT Atlantique LS2N - CNRS UMR 6004 La Chantrerie, France
}

\begin{abstract}
The ripple effect could occur when a disruption in supplier base cannot be localised and its consequences propagate downstream the supply chain (SC) adversely affecting the performance. While stress-testing of SC designs and assessment of their vulnerability to disruptions in single-echelon-single-event setting is desirable and indeed critical for some firms, modeling the ripple effect impact in multi-echelon-correlated-events systems is becoming increasingly important. Notably, ripple effect assessment in multi-stage SCs is particularly challenged by consideration of both vulnerability and recoverability capabilities at individual firms in the network. We construct a new model based on integrating Discrete-Time Markov Chain (DTMC) and Dynamic Bayesian Network (DBN) model to quantify the ripple effect. We use DTMC to model the recovery and vulnerability of suppliers. The proposed DTMC model is then equalized with a DBN model in order to simulate the propagation behavior of supplier disruption in the SC. Finally, we propose a metric that quantifies the ripple effect of supplier disruption on manufacturer in terms of total expected utility and service level. The ripple effect measure constructed is examined and tested using two case-studies. The findings suggest that our model can be of value in revealing latent high-risk paths in the SC, analysing the performance impact of both a disruption and its propagation, and prioritizing the contingency and recovery policies.
\end{abstract}

Keywords: Ripple effect, Supply disruption, Bayesian network, Supply chain resilience

\section{Introduction}

In recent years supply chains (SCs) have become exposed to disruptions due to several reasons such as the growth of global sourcing, the complex and interconnected structure of supply networks, and increasing adoption of demanddriven and lean production practices.

The unpredictability of the disruption appearance and the magnitude of the propagation of disruption consequences throughout multiple network echelons emerged with a new research stream - the ripple effect in the SC [1, 2]. The ripple effect in the SC refers to structural dynamic and describes a a downstream propagation of the disruption consequences, e.g., in form of downscaling in demand fulfilment, downstream the SC as a result of a severe disruption (or a series of disruptions). Illustrative examples of SC disruptions show that their localisation is frequently impossible due to multiple interconnections and outsourcing activities resulting in propagation of disruptions and the associated

\footnotetext{
${ }^{*}$ Corresponding author

Email addresses: mohsen.hosseini@usm.edu (Seyedmohsen Hosseini), dmitry.ivanov@hwr-berlin.de (Dmitry Ivanov), alexandre.dolgui@imt-atlantique.fr (Alexandre Dolgui)
} 
performance impact. For example, Dolgui et al. [3] reported the downstream propagation of the demand fulfillment downscaling as a result of the ripple effect.

After Japanese tsunami and earthquake in 2011, many auto manufacturing factories in England and USA forced to stop their production due to the disruption of their auto part suppliers who were located in tsunami and earthquake zone. The impact of this natural disaster was not solely limited to auto SC but also shocked electronic SCs. Sony, one of the giant electronic companies halted the production of its image sensor that used in smart cameras, including Apple iPhone. The Renesas, one of the largest electronic and the automotive microcontroller SC in the world suffered damage at its semiconductor plants following the tsunami, leading to months of delays to the global SC for auto manufacturers. It is noted that Renesas had not been stocking surplus inventory for managing risk disruption since the tsunami disaster (Reuters 2016). In a more recent natural disaster in December 2015, a severe flood hit Chennai city, one of the major auto hub with several automaker plants such as Hyundai, Ford, Renault, BMW, and Daimler. The operations of assembly, process, and manufacturing units stopped and suppliers exhausted as flood waters closed over Chennai. The lost production capacity of Hyundai and Renault was estimated roughly $0.68,0.3$ and 0.5 million units respectively (Beroe Inc. 2015). In complex SCs like automobile and electronic, a firm produces a component of a part and then passes it on to a domestic or global supplier for supplementary assembly. For example, the chip component of Wii console designed by IBM, fabbed in New York, tested in Taiwan, stocked in Hong Kong, aggregated in parts, and sent in kits to China for final assembly (EETimes [4]).

Examples discussed above show how SCs could be vulnerable to disruptions and their propagation. As such, there is a necessity for resilience planning which requires two considerations: reducing vulnerability to disruptions and enhancing recoverability, or the ability of SCs to recover timely to the desired service level. Many of automaker firms changed their SC strategies based on the lessons they learned from the Japanese tsunami. Some of these changes include multi-sourcing consideration instead of single-sourcing, collaborating with suppliers who are geographically located in a free disaster zone, building transport flexibility, and holding surplus inventory for some critical parts. In order to assess, select and successfully implement these resilience strategies, SC stakeholders need decision-support methods and models to understand and analyse the disruption risks and adverse effects associated with the risk propagation. Measuring the vulnerability of SC entities prior to disruption can aid SC as a whole to mitigate disruption quickly with minimal costs. Among SC entities, suppliers play a critical role as their disruptions can have far-reaching consequences.

One difficulty in the context of SC disruption propagation analysis is how to measure the ripple effect of a disruption on the downstream of SC entities (Scheibe and Blackhurst [5]). While stress-testing of SC designs and assessment of their vulnerability to disruptions in single-echelon-single-event setting is desirable and indeed critical for some firms, modeling the ripple effect impact in multi-echelon-correlated-events systems is becoming increasingly important. Measuring the ripple effect is challenging and has not been explored well enough. We are aware of two ripple effect metrics, the one based on supply ratio measurement [3] and the second one based on SC performance impact [6]. None of these measures, though, do not specify the behavior of SC firms (i.e., the changes in their operational states in terms of vulnerability and recovery) during the disruption and recovery process a distinctive and significant contribution made by the present study.

Our study builds on and contributes to the research on measuring the disruption impact on SC performance making substantive contributions. First, we model the disruption propagation with consideration of changes in operational states at SC nodes. Notably, ripple effect assessment in multi-stage SCs is particularly challenged by consideration of both vulnerability and recoverability capabilities at individual firms in the network. We construct a new model based on integrating Discrete-Time Markov Chain (DTMC) and Dynamic Bayesian Network (DBN) model to quantify the ripple effect. We use DTMC to model the recovery and vulnerability of suppliers. The proposed DTMC model is then equalised with a DBN model in order to simulate the propagation behavior of supplier disruption in the SC. Second, we propose a metric that quantifies the ripple effect of supplier disruption on manufacturer in terms of total expected utility and service level. The ripple effect measure constructed is examined and tested using two case-studies. The findings suggest that our model can be of value in revealing latent high-risk paths in the SC, analysing the performance impact of both a disruption and its propagation, and prioritizing the contingency and recovery policies.

The rest of this study is organised as follows: Section 2 reviews relevant previous literature. The proposed DTMC model is introduced in Section 3. Section 4 describes the equivalent DBN. Two case studies are discussed in Section 5 and finally the paper ends with findings and conclusions in Section 6. 


\section{Literature Review}

There is a growing interest in measuring the impact of disruptions on SCs but the literature of disruption management of SCs with the ripple effect consideration is very limited. For example, Burgert and Lasch [7] developed dynamic simulation model to quantify disruption costs subject to different disruption length. Their simulation model is capable of evaluating effectiveness of various responsive pricing and fulfillment rate. Sawik [8] introduced a stochastic mixed integer programming with an embedded network flow structure to find most appropriate portfolio approach to mitigate disruption. Behdani et al. [9]. Wang et al. [10] proposed a quantitative method based on design structure matrix to study the impact of structural complexity on SC disruption. Wang et al. [11] developed a petri net model to evaluate the impact of disruption on SCs service level performance.

Recent studies on modeling and measuring the impacts of a supplier disruption have mostly explored single-stagesingle-event setting rather than the impact of the ripple effect throughout the multi-stage supply networks.

The ripple effect occurs when the consequences of disruption cannot be localised or being contained to one part of SC, and cascades downstream and impact the performance of SC [2]. This impact could be increased on delivery time, lowering marginal profits, reducing stock return or even loss of market share and reputation. The ripple effect deals with low likelihood, high impact disruptive events which could potentially have long-term negative effects on SC system, which is an inverse of the bullwhip effect [1, 12, 13, 14]. Ivanov et al. [2] defined and explored the ripple effect as from disruption propagation of an initial disruption networks other SC stages in the supply, production, and distribution networks. Ivanov [5] used discrete-event simulation technique using anyLogistix software, where each structural model object is modeled as an agent to study the ripple effect on a four-stage SC. The simulation model aims to mimic how disruptions in the upstream influence the manufacturing firms and performance of downstream SC entities. Sokolov et al. [15] proposed a two-model multi-criteria approach to examine the potential ability of SCD to remain stable at the execution stage, where different operability objectives such as stability, robustness, and resilience are taken into decisions. Left ignored, however, was the impact of changes in the operational states at network nodes during the disruption and recovery. Levner and Ptuskin [16] studied the ripple effect of environmental disruptions such as emissions, wastes, recourse depletion, and effluents on SCs. The authors presented an entropy based optimization model to evaluate the economic loss caused by environmental disruption factors subject to the ripple effect. Ivanov et al. [13] examined dynamic recovery policies for time-critical supply chains in the presence of a ripple effect. The authors represented reactive recovery policies in dairy SC under to tackle ripple effect from resilience and flexibility perspectives. Ojha et al. [17] developed a Bayesian network model to evaluate supply chain risk propagation. The ripple effect of node disruption is assessed using metrics such as fragility, service level, inventory cost, and lost sales. Ivanov et al. [18] investigated the impact of the ripple effect on digital technology and Industry 4.0. The authors discussed that digitalization can contribute to enhancing ripple effect control and technology-based extensions can trigger the development of SC risk. The authors argued that additive manufacturing and Industry 4.0 are more susceptible to the external risk and ripple effect since the sourcing and production activities are influenced by digitalization.

Scheibe and Blackhurst [19] provided insights into the understudied causes propagation SCDs. The impact of propagation on SCs is then analysed based on examining the nature of disruption, structure, and dependency among SC entities, and managerial decision making. Han and Shin [20] introduced stochastic metrics to evaluate the structural robustness of SCs during under disruption propagation consideration. Disruption propagation throughout SC is measured in terms of connectivity between SC nodes. Tan et al. [21] built a discrete-event simulation model to measure the magnitude of disruption propagation based on the notion of water ripples. Their simulation model modeled disruption on both supply and demand nodes and recovery time as well. Otto et al. [22] studied the economic impacts of unpredicted production disruptions caused by natural disaster and its propagation impact throughout the global supply networks. The authors picked a case study of Japanese manufacturing firm to show the impact of manufacturing disruption can cascade on warehouses. The authors discussed that warehousing is a central option to decrease the indirect economic losses, and also redundancy in the supplier base can help to reduce the likelihood of manufacturing disruption by avoiding supply shortage. Ivanov [23] studied production-ordering systems in supply chains with the disruption risks in recovery and the influence of disruptions on production and distribution networks through discrete event simulation, performed on anyLogistix software. His findings show that the inventory control system will fail to return to its normal condition if there is high backlog accumulating during the disruption period. Additionally, the author found that discontinuing of contingency plans after capacity restoration could result in destabilization of the inventory management system. Ivanov et al. [24] developed a bi-objective hybrid linear programming-system dynamic 
model to analyse the ripple effect impact on closed-loop supply chains. The authors examined the impact of different recovery strategies on return costs in the upstream SC parts. Ivanov et al. [25] proposed a dynamic bi-objective multistage multi-product programming model to study the impact of different resilience actions on SC performance and distribution replanning. Ivanov et al. [26] developed a maximal flow LP based optimization model to study operational distribution replanning under ripple effect. Their proposed model describes SC as a non-stationary dynamic system along with a linear programming model. Their model is capable of ripple effect and structure dynamics which help to consider different replanning scenarios in the case of disruptions. The readers are referred to Ivanov et al. [27] for a comprehensive review of the research stream on the impact of ripple effect in the SC.

While studies have established the salience of vulnerability and recoverability in analysing the ripple effect, including successful risk mitigation practices, little attention has been directed to their interplay in the settings with disruption propagation. In this paper we first present a DTMC model that captures supplier capacity downgrading in the presence of a disruption and the supplier capacity recovery. Notably, we contribute to literature by conceptualizing an extended view of modeling the firms operational dynamics during the disruption and recovery by including gradual operability degradation and recovery. This approach appears to be more relevant in a practical decision environment as opposite to the more common view of either completely disrupted or fully operational network capacities, i.e. $0-1$ setting. The proposed model, in fact, mimics the vulnerability and recoverability of suppliers, two important preconditions for assessing supply base risks and identifying critical exposure points. The DBN equivalent of Markov model is then proposed to examine the propagation impact of a supplier disruption in a dynamic (time-dependent) environment that in turn, feeds an explicit quantification of the ripple effect. The combination of DTMC and DBN is unique in the literature and mimics the complexity of business reality affording more realistic applications to making SC design, sourcing, and resilience management decisions.

\section{Proposed Markov chain model}

This section presents a Markov chain model to simulate the behavior of a supplier in the case of disruption. Consider a supplier whose behavior can be described with the help of three states (Figure 1). In these states, a supplier can fully absorb the shocks of disruption without losing its capacity, named as a fully operational state, or when supplier loses all its capacity due to the severity and magnitude of disruption, named as fully disrupted state, or when supplier partially lose its capacity, named semi-disrupted state. Although multiple semi-disrupted states can be taken into account but for simplification purpose and without loss of generality, we consider single semi-disrupted state which represents the condition when a supplier loses its $50 \%$ of its capacity in the presence of disruption. The proposed Markov chain consists of three discrete states 0,1 , and 2 to capture the capacity losses of supplier after the realization of disruption. These three states represent the capacity of the supplier in fully operational, semi-disrupted and fully disrupted states respectively. The Markov chain model is represented in Figure 1.

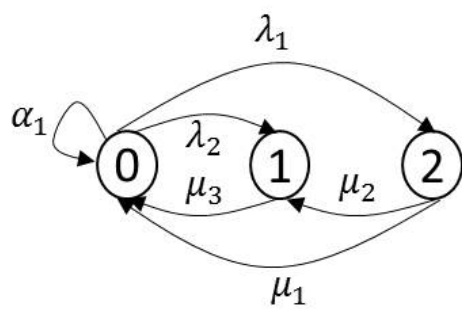

Figure 1: Discrete time Markov chain model for supplier capacity disruption.

Let's assume that supplier is under operating normal condition prior to a disruption. If a disruption occurs, a supplier could absorb the shocks of disturbance without losing of its supply capacity. This case is modeled using a self-loop in state 0, known as absorbing or operational state. On the other hand, the supplier might lose 50\% of its capacity after a disruption occurs, so this case is captured by an outgoing arc from state 0 to state 1 . Under the worst case scenario, a supplier may lose all its operational capacity if a disaster with high severity and degree of exposure occurs. Our model captures this scenario by a forward transition from state 0 to sate 2 . Now, let's assume that a 
supplier is semi-disrupted, and is in state 1 in time $t$. That supplier can restore its lost capacity by backward transition from state 1 in time $t$ to state 0 in time $t+1$. We assume that disrupted supplier starts to restore its lost capacity after disruption is realized. Hence, there is no transition from state 1 to state 2 . Finally, consider the case where the supplier is fully disrupted in time $t$, the supplier can restore all its lost capacity in time $t+1$, captured by an incoming arrow from state 2 to state 0 , or $50 \%$ of lost capacity can be restored in time $t+1$ and the rest in time $t+2$, so this case is captured by two outgoing arrows from state 2 to state 1 and from state 1 to state 0 . It is notable that $\pi_{0}, \pi_{1}$ and $\pi_{2}$ denotes the probability of the supplier being at state 0,1 and 2 respectively. The transition rate of going from a state to other state is represented by $\alpha_{1}, \lambda_{1}, \lambda_{2}, \mu_{1}, \mu_{2}$, and $\mu_{3}$ as illustrated in Figure 1 . The balance questions and the norming equation of the model is represented in equations 1-4. By solving the system of equations given below, the probability that a supplier is on state 0,1 and 2 can be obtained. By knowing the probability of each state, supplier can have a better understanding about its vulnerability and make an efficient resilience plan to mitigate disruption.

$$
\begin{aligned}
\left(\alpha_{1}+\mu_{1}+\mu_{3}\right) \pi_{0} & =\lambda_{1} \pi_{2}+\lambda_{2} \pi_{1} \\
\left(\lambda_{2}+\mu_{2}\right) \pi_{1} & =\mu_{3} \pi_{0} \\
\lambda_{1} \pi_{2} & =\mu_{1} \pi_{0}+\mu_{2} \pi_{1} \\
\pi_{0}+\pi_{1}+\pi_{2} & =1
\end{aligned}
$$

\section{Equivalent Dynamic Bayesian network model}

\subsection{Bayesian network theory}

BNs are recognised as a powerful methodology for managing risk assessment, uncertainty, and decision making. BNs are capable of combining expert knowledge with historical data to quantify risk exposure by capturing the causes and effects using graphical representation. $\mathrm{BN}$ is a probabilistic directed acyclic graphical model that is structured based on Bayes' theorem. BNs have been widely used to deal with risk management problems [28, 29, 30, 31, 32, 33]. From the mathematical point of view, BNs can be modeled as acyclic directed graphs with a set of nodes (variables), represented by $V=\left\{X_{1}, X_{2}, \ldots, X_{n}\right\}$, and a set of arcs (edges) whose structure determines the relationship between nodes. An outgoing arc from $X_{i}$ to $X_{j}$ represents that the value of variable $j$ depends on the value of $X_{i}$, then $X_{i}$ and $X_{j}$ are called parent and child node respectively. Three different types of node exist in BN: (i) nodes without a parent node are called root nodes, (ii) nodes without a child node are called leaf nodes, and (iii) nodes with parent and child nodes are called intermediate nodes [28]. The joint probability of all variables (nodes) can be written using chain rule as the product of the conditional probability of each node as follows:

$$
P\left(X_{1}, X_{2}, \ldots, X_{n}\right)=\prod_{i=1}^{n} P\left(X_{i} \mid X_{1}, X_{2}, \ldots, X_{i-1}\right)
$$

For example, consider the BN model with 4 variables depicted in Figure 2, $X_{1}$ is root node, $X_{2}$ and $X_{3}$ are intermediate nodes and $X_{4}$ is leaf node.

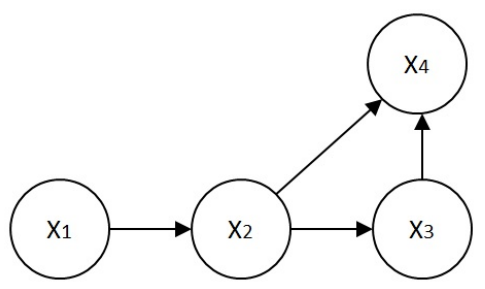

Figure 2: An illustrative BN with 4 variables (nodes)

The conditional probability is used in BNs to captures the causal relationship among variables. The full joint probability distribution (JPD) of the BN models can be viewed as a representation of the structure of the BN and dependencies among variables. In example illustrated above, prior probability of $P\left(x_{1}\right)$ and conditional probabilities of $P\left(x_{2} \mid x_{1}\right), P\left(x_{3} \mid x_{2}\right)$ and $P\left(x_{4} \mid x_{2}, x_{3}\right)$ must be defined. The JDP of BN model in Figure 2 is calculated as: 


$$
P\left(x_{1}, x_{2}, x_{3}, x_{4}\right)=P\left(x_{1}\right) P\left(x_{2} \mid x_{1}\right) P\left(x_{3} \mid x_{2}\right) P\left(x_{4} \mid x_{2}, x_{3}\right)
$$

In BNs, each variable is associated with a conditional probability table (CPT), which determines the probability of the occurrence of a realization of a variable given the values of other variables [28]. It is notable that CPT can express the conditional probability between two variables.

The JDP can be used to calculate the probability of individual variables in a BN. Suppose that we are interested in calculating $X_{2}$, then $P\left(X_{2}\right)$ can be written with equation 7 .

$$
P\left(x_{2}\right)=\sum_{x_{1}, x_{3}, x_{4}} P\left(x_{1}\right) P\left(x_{2} \mid x_{1}\right) P\left(x_{3} \mid x_{2}\right) P\left(x_{4} \mid x_{2}, x_{3}\right)
$$

The equation above can be rewritten to the one shows in equation 7 below using marginalization technique. Marginalization is a distributive operation over a combination of local joint probabilities, meaning that we can marginalize the global probability by marginalizing local CPTs [34].

$$
P\left(x_{2}\right)=\left(\sum_{x_{1}} P\left(x_{1}\right)\left(\sum_{x_{3}} P\left(x_{3} \mid x_{2}\right)\left(\sum_{x_{4}} P\left(x_{4} \mid x_{2}, x_{3}\right)\right)\right)\right)
$$

To demonstrate the impact of supplier disruption using BN, let's consider a simple example. BN model consists of two suppliers 1 and 2 denoted by $s_{1}, s_{2}$ and a single manufacturer denoted by M. It is clear that a manufacturer could potentially fail to continue its operations if suppliers fail to supply raw materials. As such, the disruption likelihood of manufacture depends on the disruption of suppliers. This casual dependency between manufacture and its suppliers is captured by incoming arcs from suppliers to manufacture as illustrated in Figure 3.

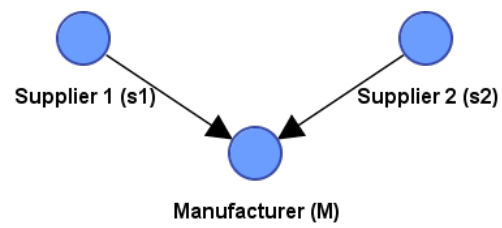

Figure 3: A simple BN with two suppliers and a single manufacturer.

The prior disruption probability of each supplier is assumed in the example showing in Figure 4 to $4 \%$, meaning that with the likelihood of $4 \%$ each supplier may fail to supply raw material to the manufacturer. Disruption on suppliers can induce disruption at manufacture with a specific probability. The conditional probability of manufacture disruption given disruption of each supplier is listed in Table 1. For example, the disruption probability of manufacture is 0.86 if supplier 1 is disrupted and supplier 2 is operational. The marginal distribution probability of manufacture is calculated based on the prior probability of suppliers unconditional probability of manufacture and suppliers as represented in equation 9.

Table 1: Condition probability table (CPT) of manufacturer disruption

\begin{tabular}{lcccc}
\hline Supplier 1 & \multicolumn{2}{c}{ Disrupted } & \multicolumn{2}{c}{ Operational } \\
\hline Supplier 2 & Disrupted & Operational & Disrupted & Operational \\
\hline Manufacturer disrupted & 0.99 & 0.86 & 0.89 & 0.02 \\
Manufacturer operational & 0.01 & 0.14 & 0.11 & 0.98 \\
\hline
\end{tabular}




$$
\begin{aligned}
P(\text { M disrupted }) & =\sum_{s_{1}, s_{2}} P\left(M \text { disrupted } \mid s_{1}, s_{2}\right)= \\
& P\left(M \text { disrupted } \mid s_{1}=\text { disrupted, } s_{2}=\text { disrupted }\right) \times P\left(s_{1}=\text { disrupted }\right) \times P\left(s_{2}=\text { disrupted }\right)+ \\
& P\left(M \text { disrupted } \mid s_{1}=\text { operational, } s_{2}=\text { disrupted }\right) \times P\left(s_{1}=\text { operational }\right) \times P\left(s_{2}=\text { disrupted }\right)+ \\
& P\left(M \text { disrupted } \mid s_{1}=\text { disrupted, } s_{2}=\text { operational }\right) \times P\left(s_{1}=\text { operational }\right) \times P\left(s_{2}=\text { disrupted }\right)+ \\
& P\left(M \text { disrupted } \mid s_{1}=\text { operational, } s_{2}=\text { operational }\right) \times P\left(s_{1}=\text { operational }\right) \times P\left(s_{2}=\text { operational }\right) \\
& =(0.99 \times 0.04 \times 0.04)+(0.89 \times 0.96 \times 0.04)+(0.86 \times 0.04 \times 0.96)+(0.02 \times 0.96 \times 0.96)=8.72 \%
\end{aligned}
$$

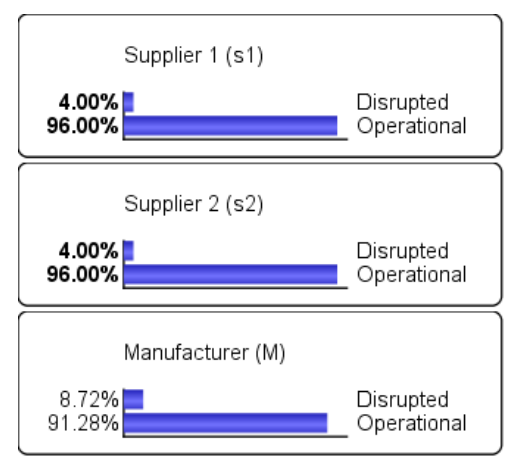

Figure 4: Probability distribution of suppliers and manufacturer.

\subsection{Dynamic Bayesian network}

DBN is an extension of static BN where the temporal dimension is taken into account by unrolling the network on the desired number of time steps (i.e., by duplicating the network for each time step). DBN can be used to model stochastic process under a dynamic environment. Some applications of DBN in the field of risk assessment can be found in $[35,36,37,38,39]$. DBN is an appropriate methodology to capture the ripple effect of supplier's disruption as the ripple effect is stochastic and dynamic in nature. To simplify the modeling process of the ripple effect, two primary assumptions are taken into account:

- The system is time invariant, i.e. the conditional probability table (CPT) does not evolve with respect and the topology of BN remains the same over different time periods.

- The process is Markovian, i.e. the probability distribution of the state at time $t-1$ depends on only the state at time $t$, not the sequence of events that precede it.

Based on the assumptions mentioned above, the DBN is defined as an extension of static BN with different time-slices. A representation of a simple DBN is depicted in Figure 5. It shows the relationship between variables in time $t$ and $t+1$. As is illustrated in Figure 5, the probability distribution of supplier at time $t+1$ depends on the its probability distribution at time $t$. The joint probability distribution of DBN under dynamic environment can be represented as:

$$
P\left(X_{1, t}, X_{2, t}, \ldots, X_{n, t}\right)=\prod_{t=1}^{T} \prod_{i=1}^{N} P\left(X_{i, t} \mid X_{1, t}, X_{2, t}, \ldots, X_{i-1, t}\right)
$$




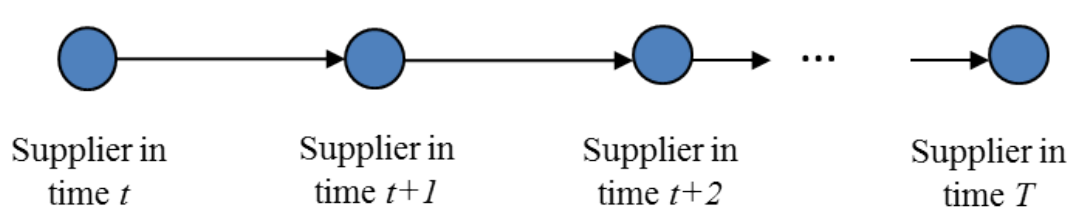

Figure 5: Dynamic Bayesian network of supplier with $T$ time slices.

\subsubsection{Transition probability}

A key challenge in the structure of DBN is on how to define transition probability as the status of variables change over time. The disruption and recovery rate of a supplier is assumed to be exponential distribution [40].

Based on the Markov transition matrix and exponential distribution assumption of disruption rate and recovery rate, the transition probability of supplier disruption is obtained and illustrated in Table 2. Given that the disruptions are uniformized (discrete) process with disruption rate $q$, and $\mathrm{N}(\mathrm{t})$ is the total number of transitions in $[0, \mathrm{t}]$, the probability that the number of transition in $[0, \mathrm{t}]$ is $k$ is:

$$
P[N(t)=k]=e^{-q_{i j} t} \frac{\left(q_{i j} t^{k}\right)}{k !}
$$

$k$ step transition probability with disruption rate of $q_{i j}$ from state $i$ to state $j$ is:

$$
T_{i j}^{k}\left(q_{i j}\right)=P\left[X_{k}\left(q_{i j}\right)=j \mid X_{0}\left(q_{i j}\right)=i\right]
$$

The Eq. (12) can be interpreted as Eq. (13):

$$
P_{i j}(t)=e^{-q_{i j} t} \sum_{k=0}^{\infty} \frac{\left(q_{i j} t\right)^{k}}{k !} T_{i j}^{k}\left(q_{i j}\right)
$$

Eq. (13) can be rewritten in the form of conditional probability as shown in Eq.(14):

$$
P_{i j}(t)=\sum_{k=0}^{\infty} P\left[X_{k}\left(q_{i j}\right)=j \mid X_{0}\left(q_{i j}\right)=i, N(t)=k\right] P[N(t)=k]
$$

The transition probability from state $i$ to state $j$ with disruption rate of $q_{i j}$ given in Eq. (14) can be obtained by expanding $P_{i j}(t)$ to first order with fixed step $h=\Delta t$. So $P_{i j}$ can be calculated as:

$$
P_{i j}=q_{i j} \Delta t \quad \forall(i \neq j)
$$

For example the transition probability from operational state to semi-disrupted and from operational state to fully disrupted state with disruption rate of $q_{02}$ is $\lambda_{1} \Delta t$.

According to the Markov chain model, the transition matrix of supplier is given by Eq. (16):

$$
P=\left[\begin{array}{ccc}
1-\left(\lambda_{1}+\lambda_{2}\right) \Delta t & \lambda_{2} \Delta t & \lambda_{1} \Delta t \\
\mu_{3} \Delta t & 1-\mu_{3} \Delta t & 0 \\
\mu_{1} \Delta t & \mu_{2} \Delta t & 1-\left(\mu_{1}+\mu_{2}\right) \Delta t
\end{array}\right]
$$

Based on transition matrix and exponential distribution of disruption and recovery rate, the CPT of state transition is obtained and represented in Table 2. 
Table 2: Conditional probability table (CPT) of three state transitions.

\begin{tabular}{lccc}
\hline$t$ & $t+\Delta t$ & & \\
\hline & operational & semi-disrupted & fully disrupted \\
\hline operational & $e^{-\left(\lambda_{1}+\lambda_{2}\right) \Delta t}$ & $\frac{\lambda_{2}}{\lambda_{1}+\lambda_{2}}\left(1-e^{-\left(\lambda_{1}+\lambda_{2}\right) \Delta t}\right)$ & $\frac{\lambda_{1}}{\lambda_{1}+\lambda_{2}}\left(1-e^{-\left(\lambda_{1}+\lambda_{2}\right) \Delta t}\right)$ \\
semi-disrupted & $e^{-\mu_{3} \Delta t}$ & $1-e^{\mu_{3} \Delta t}$ & 0 \\
fully disrupted & $\frac{\mu_{1}}{\mu_{1}+\mu_{2}}\left(1-e^{-\left(\mu_{1}+\mu_{2}\right) \Delta t}\right)$ & $\frac{\mu_{2}}{\mu_{1}+\mu_{2}}\left(1-e^{-\left(\mu_{1}+\mu_{2}\right) \Delta t}\right)$ & $e^{-\left(\mu_{1}+\mu_{2}\right) \Delta t}$ \\
\hline
\end{tabular}

\section{Case studies}

\subsection{Case study 1}

Let's consider a single supplier with three states of operational, semi-operational and fully disrupted. This simple case study examplifies the scenario under which our modeling methodology would be deployed. The proposed DBN is used to predict the dynamic characteristic of supplier's disruption over eight time-slices periods. Given that $\lambda_{1}=0.07$, $\lambda_{2}=0.11, \mu_{1}=0.27, \mu_{2}=0.41, \mu_{3}=0.54$. The CPT of supplier from time $t$ to time $t+1$ can be represented in Table 3.

Table 3: Conditional probability table (CPT) of three state transitions.

\begin{tabular}{lccc}
\hline$t$ & $t+\Delta t$ & & \\
\hline & operational & semi-disrupted & fully disrupted \\
\hline operational & 0.835 & 0.101 & 0.064 \\
semi-disrupted & 0.583 & 0.417 & 0 \\
fully disrupted & 0.204 & 0.554 & 0.242
\end{tabular}

The proposed DBN is used to predict the dynamic behavior of a supplier and how the presence of disruption can propagate over eight different time-slices, as illustrated in Figure 6. The CPT represented in Table 3 is used to capture the conditional causality between every two consecutive time slices from $t=2$ to $t=8$. The prior probability of supplier being operational, semi-disrupted and fully disrupted at time slice $t=1$ is $88 \%, 3 \%, 9 \%$ respectively.

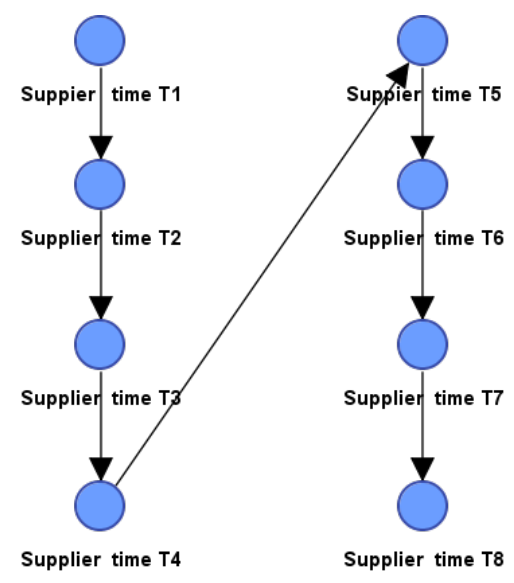

Figure 6: DBN disruption modeling of supplier over 8 time slices.

The posterior probability of supplier for each time slice is represented in Figure 7. From this figure, the probability of supplier being operational is $88 \%$ in $t=1$ and reduces to $77.06 \%$ in $t=2$. This Figure shows how the distribution probability of supplier changes over 8 time periods. Seen from Figure 7, the probability of supplier being operational drops by $12.43 \%$ from $t=1$ to $t=2$ and this probability increases slightly from $t=4$ to $t=8$. Although the distribution probability of supplier can be obtained by DBN over different time periods, however, we are interested 
to see how these probabilities are impacted, particularly the operational probability of supplier at final time period $(t=8)$ when a disruption occurs in the initial time period $(t=1)$. In other words, we would like to evaluate the impact of disruption propagation on target node $(t=8)$ when disruption occurs at early stages (e.g., $t=1, t=2)$. The next section discus disruption propagation analysis in more details.

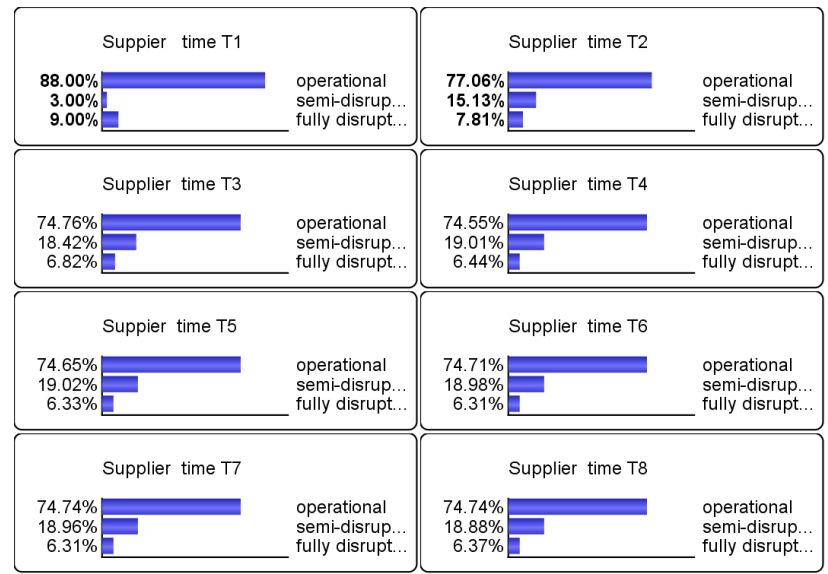

Figure 7: Posterior disruption probability of supplier in time $t=2$ to $t=7$ and prior probability of supplier in $t=1$.

Disruption modeling is stochastic and dynamic in nature, meaning that it involves occupance uncertainty and the impact of disruption does not limit to only a single time period and may propagate over several time periods. Hence, it is critical to analyse the dynamic impact of disruption. Causal inference is a powerful feature of BN that allows us to make an observation on a node (variable) based on some evidence and see the impact of that observation propagates throughout the network. Here, we would like to see how a disruption occurrence can impact the operational state of supplier over time periods. As such, we made an observation on the supplier in time $t=1$ by assuming that supplier is fully disrupted in $t=1$ and propagates the impact of this evidential reasoning over the BN. Figure 8 illustrates causal inference on supplier in time $t=1$ by setting the probability of supplier being disrupted in $t=1$ to $100 \%$ and updating the marginal probability distribution (MPD) of unobserved variables. There are different algorithms that can be used to update the MPD of an unobserved variable such as the junction tree algorithm (JTA). The JTA has been used in this study. The readers may refer to Zheng et al. [41] for more details of JTA. From Figure 8, the probability of supplier being operational in $t=8$ reduces from $74.74 \%$ in baseline model to $68.56 \%$ when supplier is fully disrupted in $t=1$. It is noteworthy to mention that other observations can be made in each time period through causal inference analysis but to avoid being lengthy we limit the causal inference analysis to only one observation in the first time slice.

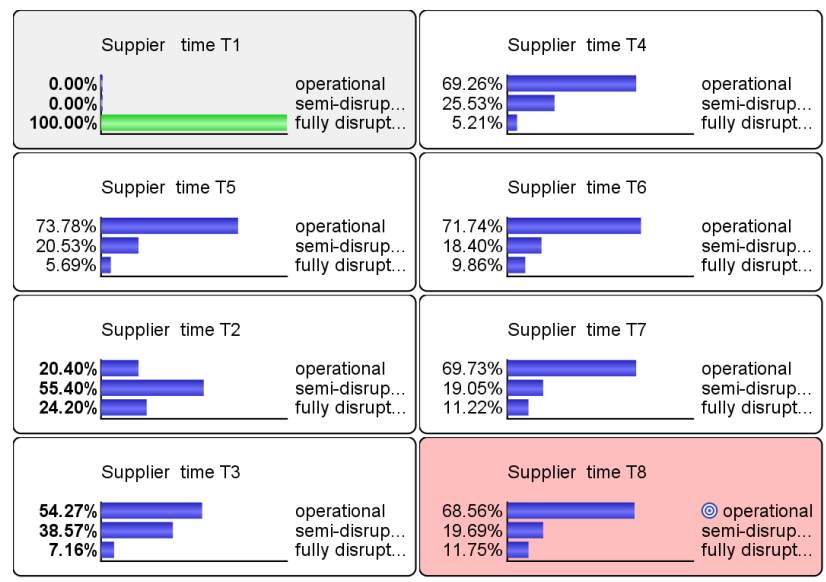

Figure 8: Disruption modeling of supplier in time $t=1$ using causal inference. 


\subsection{Case study 2}

The second case study is a two-stage supply chain of RFID blocking bi-fold wallet with a single manufacturer and two suppliers. The two suppliers supply leather and RFID blocking chip weekly. The DBN represented of this supply chain is illustrated in Figure 9. As it can be seen from Figure 9, the manufacturer in the initial time slice, $t=1$, conditions on suppliers 1 and 2 . The operations of manufacturers in time $t+1, t+2$ and $t+3$ depends on its operations in its previous time $t, t+1$ and $t+2$ respectively. The suppliers supply by the end of each week and supplied materials are used by manufacturers by the beginning of next week. As such, the operations of manufacture in its current time depends on the condition of suppliers in its previous time. The distribution probability of supply chain entities is illustrated in Figure 10.

\subsubsection{Causal inference analysis}

In this section, we are interested to assess the dynamic disruption impact of supplier on manufacturers. As such, three disruption scenarios are defined. Scenarios 1 and 2 that suppliers 1 and 2 are assumed to be fully disrupted separately in the first time period and scenario 3 which both suppliers are assumed to be disrupted. Scenario 3 can occur due to different reasons such as local natural disasters if both suppliers are located in the same region. The results of these three disruption scenarios on manufacturers over three time periods are depicted in Figure 11. As it can be seen from Figure 11, disruption of supplier 1 has a higher impact on the probability of operations of manufacturers in compared with supplier 2. It is observed that the probability of operations of the manufacturer in time $t+1$ reduces from $92.84 \%$ in baseline model to $71.69 \%$ in scenario 1 when supplier 1 is fully disrupted, while this probability reduces to $73.24 \%$ when supplier 2 is fully disrupted. This probability reduces to $8 \%$ when both suppliers are fully disrupted in scenario 3.

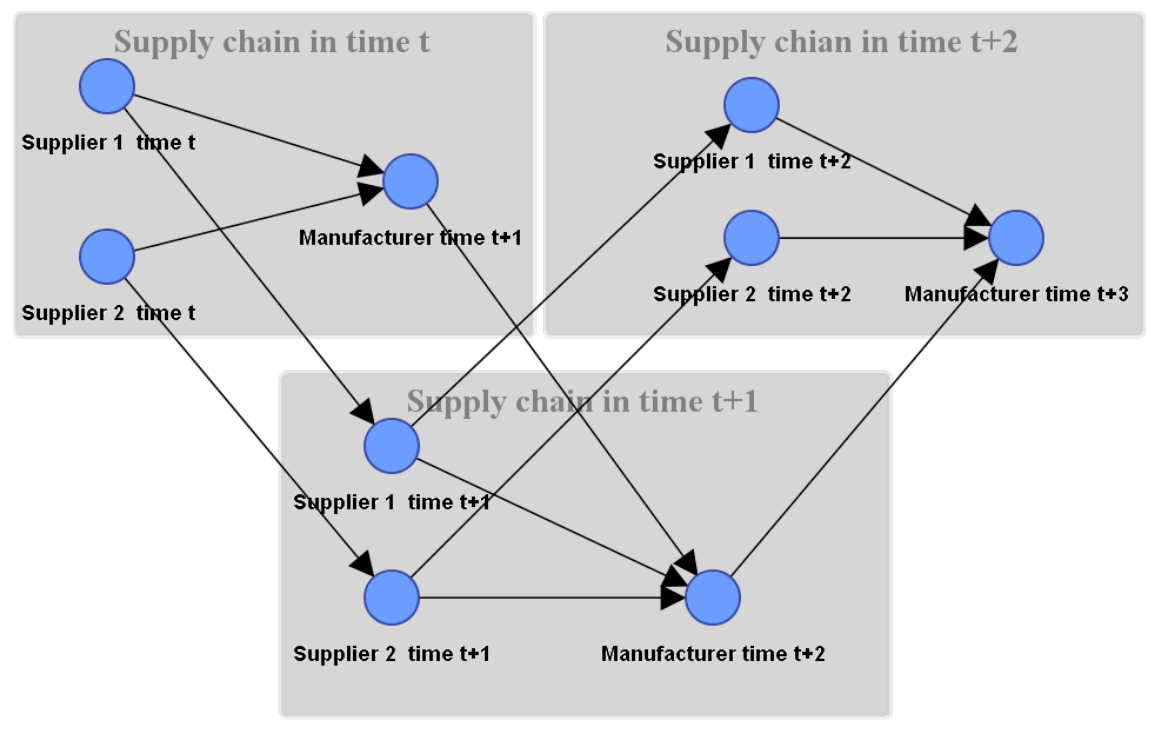

Figure 9: The DBN of two-stage supply chain over three time slices. 


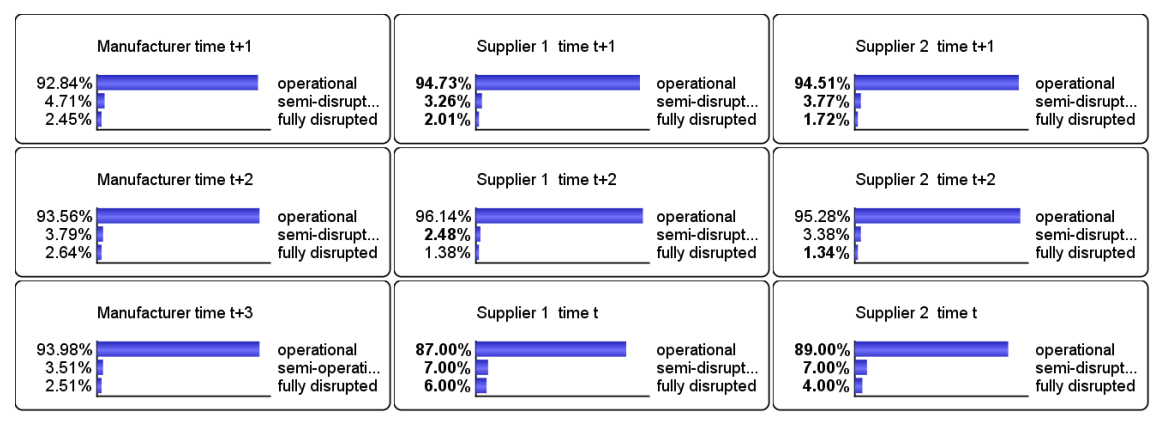

Figure 10: The distribution probabilities of supply chain entities over three time slices.

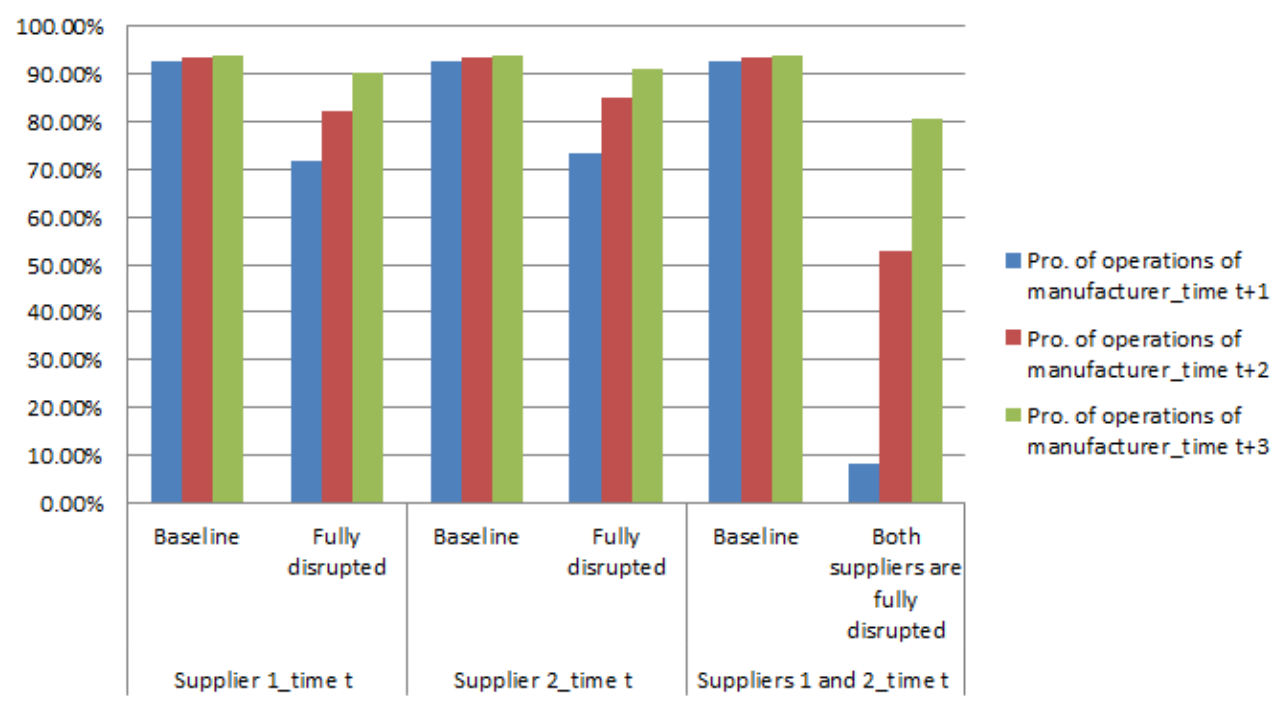

Figure 11: The distribution probabilities of supply chain entities over three time slices.

\subsubsection{Inference influence on target node}

In this case study, we assume that the manufacturer variable in time $t+3$ is the target node (interest node). We perform an influence analysis to see the sensitivity of the target node with respect to other variables (nodes). Figure 12 represents the probability that target node is operational (operational state of the target node is 100\%) given suppliers and manufacturer in different time periods. For example, the probability of manufacturer in time $t+3$ being operational given manufacturer is being operational in time $t+2$ is $95.50 \%$, while this probability changes to $88.59 \%$ and $47.95 \%$ when the manufacturer in time $t+2$ is semi-disrupted and fully disrupted respectively. The probability of manufacturer being operational in time $t+3$ given three states of the manufacturer in time $t+2$ is illustrated on the upper left corner of Figure 12. 


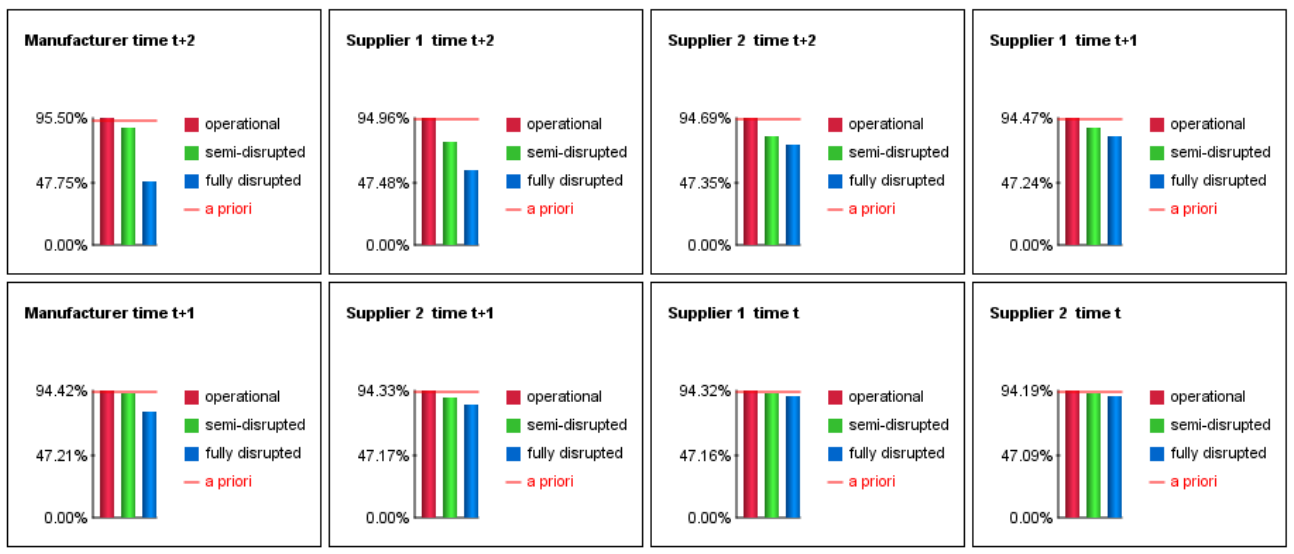

Figure 12: Influence analysis on target node (manufacturer in time $t+3$ ).

\subsection{Ripple effect modeling}

As we discussed earlier, ripple effect occur when the impact of disruption on upstream of the supply chain cannot be localised and propagates downstream and negatively impact supply chain performances such as cost, service level and lead time. Here, we borrow expected utility theory and decision trees to in order model the ripple effect. Let's consider a supplier variable with three states: fully disrupted, semi-disrupted and operational, and the probability of each state is assumed to be known. The service level of supplier highly depends on its three above-mentioned states. Supplier's service level (SL) may vary depending on whether the supplier is operational, semi-disrupted or fully disrupted. As such, SL variable conditions on supplier's status from the BN standpoint. It is assumed that the SL variable includes three states of Low, Medium, and High, meaning that probability of not having a stock-out could be either on Low, Medium or High state depending on the state of the supplier. There is a probability associated with the state of SL that is shown in Table 4. We assume that the SL of the supplier is Low when the probability of having no stock-out falls below $80 \%$ and is High when the probability of service level is greater or equal to $93 \%$. Finally, the SL of the supplier is considered to be Medium when it falls between [80\%, 93\%) interval.

Table 4: Linguistic variable of supplier's service level.

\begin{tabular}{lc}
\hline Linguistic & SL percentage \\
\hline Low & $S L<80 \%$ \\
Medium & $80 \% \leq S L<93 \%$ \\
High & $S L \geq 93 \%$ \\
\hline
\end{tabular}

Let's consider a supplier in which the probability of its being operational, semi-disrupted and fully disrupted is 0.75 , 0.13 and 0.12 as represented in Figure 13. The probability of SL being Low, Medium or High depends on the operations of the supplier. For example, the probability of SL being Low given supplier is operational, P(Low SL I operational) is 0.03 as can be seen from Figure 13. There is a utility associated with each service level state (Low, Medium, High), denoted by $U_{1}, U_{2}$, and $U_{3}$ respectively. In the example given in Figure 13, the $U_{1}, U_{2}, U_{3}$ for Low, Medium and High service level is $-5,000,-3,000$ and 5,000 respectively. The Expected Utility (EU) for state of Low, $E U_{\text {Low }}$, is calculated by Eq. (17). The EU for High and Medium states, $E U_{\text {Medium }}$ and $E U_{\text {High }}$ can be calculated similarly.

$$
\begin{aligned}
E U_{\text {Low }}= & {\left[P(\text { Operational }) \times P(\text { Low SL } \mid \text { Operational }) \times U_{1}\right]+} \\
& {\left[P(\text { Semi-disrupted }) \times P(\text { Low SL } \mid \text { Semi-disrupted }) \times U_{1}\right]+} \\
& {\left[P(\text { Fully disrupted }) \times P(\text { Low SL } \mid \text { Fully disrupted }) \times U_{1}\right]=-604.5 }
\end{aligned}
$$


The total expected utility (TEU) of supplier, $T E U_{\text {supplier }}$ is calculated as the sum of expected utility of supplier for three states of Low, Medium and High as represented in Eq. 18.

$$
T E U_{\text {supplier }}=E U_{\text {Low }}+E U_{\text {Medium }}+E U_{\text {High }}
$$

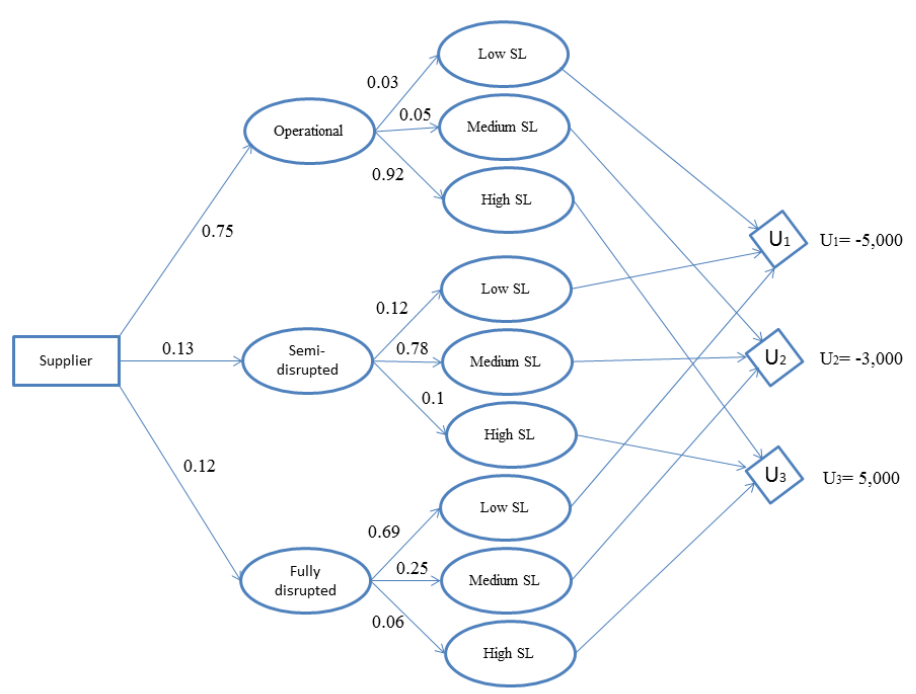

Figure 13: Decision tree containing supplier stats, service level states and utility nodes.
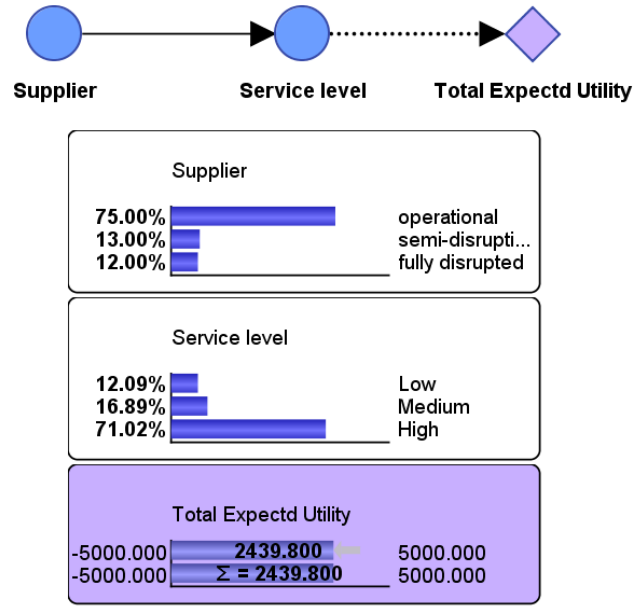

Figure 14: Total Expected Utility (TEU) of supplier.

The total expected utility of supplier, $T E U_{\text {supplier }}$ is 2439.8 , as can be seen from Figure 14. It is notable that Figure 14 represents the causal diagram of TEU for only a single time period, while the TEU of supplier over a time horizon can be obtained by the sum of TEU of that supplier for each time period. Furthermore, similar TEU can be calculated for the manufacturer.

To develop a generic metric to model ripple effect, let's consider a two-stage supply chain with $m$ suppliers, $n$ manufacturers over $T$ time horizons. Given notations below, the TEU for all suppliers in a supply chain can be calculated by Eq. 19 . 


\section{Nomenclature}

$i=1,2, \ldots, m$ set of suppliers

$j=1,2, \ldots, n$ set of manufacturers

$k=1,2,3$ state of supplier/manufacturer (operational, semi-disrupted, fully disrupted)

$l=1,2,3$ state of supplier's/manufacturer's service level (Low, Medium, High)

$t=1,2, \ldots, T$ set of time periods

$p_{i k l t} \quad$ probability that the service level (SL) of supplier $i$ with is on state $l$ in time period $t$ knowing that supplier $i$ is on state $k$

$p_{i k t} \quad$ probability that supplier $i$ is on state $k$ in time period $t$

$p_{j k l t} \quad$ probability that the service level (SL) of manufacturer $j$ with is on state $l$ in time period $t$ knowing that manufacturer $j$ is on state $k$

$p_{j k t} \quad$ probability that manufacturer $j$ is on state $k$ in time period $t$

$U_{i k l t} \quad$ expected utility associated with service level state $l$ of supplier $i$ when supplier $i$ is on state $k$

$U_{j k l t} \quad$ expected utility associated with service level state $l$ of manufacturer $j$ when manufacturer $j$ is on state $k$

$$
T E U_{\text {suppliers }}=\sum_{t=1}^{T} \sum_{i=1}^{m} \sum_{k=1}^{3} \sum_{l=1}^{3} p_{i k t} \times p_{i k l t} \times U_{i k l t}
$$

The TEU of all manufacturers over $T$ time horizons can be obtained by equation below:

$$
T E U_{\text {manufacturers }}=\sum_{t=1}^{T} \sum_{j=1}^{n} \sum_{k=1}^{3} \sum_{l=1}^{3} p_{j k t} \times p_{j k l t} \times U_{j k l t}
$$

Finally, the TEU of supply chain is obtained by:

$$
T E U_{\text {supply chain }}=\sum_{t=1}^{T} \sum_{i=1}^{m} \sum_{k=1}^{3} \sum_{l=1}^{3} p_{i k t} \times p_{i k l t} \times U_{i k l t}+\sum_{t=1}^{T} \sum_{j=1}^{n} \sum_{k=1}^{3} \sum_{l=1}^{3} p_{j k t} \times p_{j k l t} \times U_{j k l t}
$$

\subsection{Ripple effect modeling of suppliers}

Let's consider the DBN model of the two-stage supply chain that is illustrated in Figure 9. We extended this DBN model by incorporating SL and utility of manufacturers for each time period as represented in Figure 15 . The distribution probability of SL and utility of manufacturer and suppliers is shown in Figure 16. Here, we would like to simulate the ripple effect of disruption that occurs on suppliers 1 and 2 and their propagation impact on manufacturer over three time periods. To do so, we define three scenarios: Scenario 1 where only supplier 1 is assumed to be fully disrupted, scenario 2 where only supplier 2 is fully disrupted and finally scenario 3, where both suppliers 1 and 2 are fully disrupted. In Scenario 1, we made an observation on supplier 1 in time $t$ by making an assumption that supplier 1 is fully disrupted and propagate the impact of this observation throughout the BN by updating the marginal probabilities of all the unobserved variables. Here, we are interested to analyse the dynamic impact of the ripple effect of supplier 1 and supplier 2 separately by scenario 1 and 2 on the manufacturer in time periods $t+1, t+2, t+3$. Note that scenario 3 simulates the ripple effect disruption of both suppliers simultaneously on manufacture.

The simulation results of the ripple effect for supplier 1 and supplier 2 are represented in Figure $17 \mathrm{a}$ and $17 \mathrm{~b}$ respectively and the ripple effect of simultaneous disruption on suppliers 1 and 2 is illustrated in Figure 18. As illustrated in Figure 17a, the expected utility of the manufacturer in time $t+1$ reduces from 716.424 in the baseline model to 418.899 when supplier 1 is disrupted in time $t$. The expected utility of manufacturer then increases to 567.964 and 
678.857 in time period $t+1$, and $t+2$ respectively as the SL of manufacturer recovers its SL over the time. Scenario 1 shows that disruption on the first supplier reduces the probability of service level of manufacturer being High from $81.29 \%$ (baseline model, Figure 16) to $64.09 \%$ (scenario 1, Figure 17a), while this probability changes to $65.26 \%$ when supplier 2 is fully disrupted (scenario 2, Figure 17b). From Figure 17, it can be seen that the TEU of manufacture is 1670 and 1730 under scenario 1 and scenario 2 respectively. This implies that the disruption of supplier 1 could have a higher negative impact on manufacture compared with supplier 2 in terms of TEU and SL measures. Finally, the TEU and SL measure of manufacturers dynamically represented in Figure 18 when both suppliers are simultaneously fully disrupted. From Figure 18, it can be seen that the TEU and SL of manufacture reduce significantly when both suppliers disrupted.

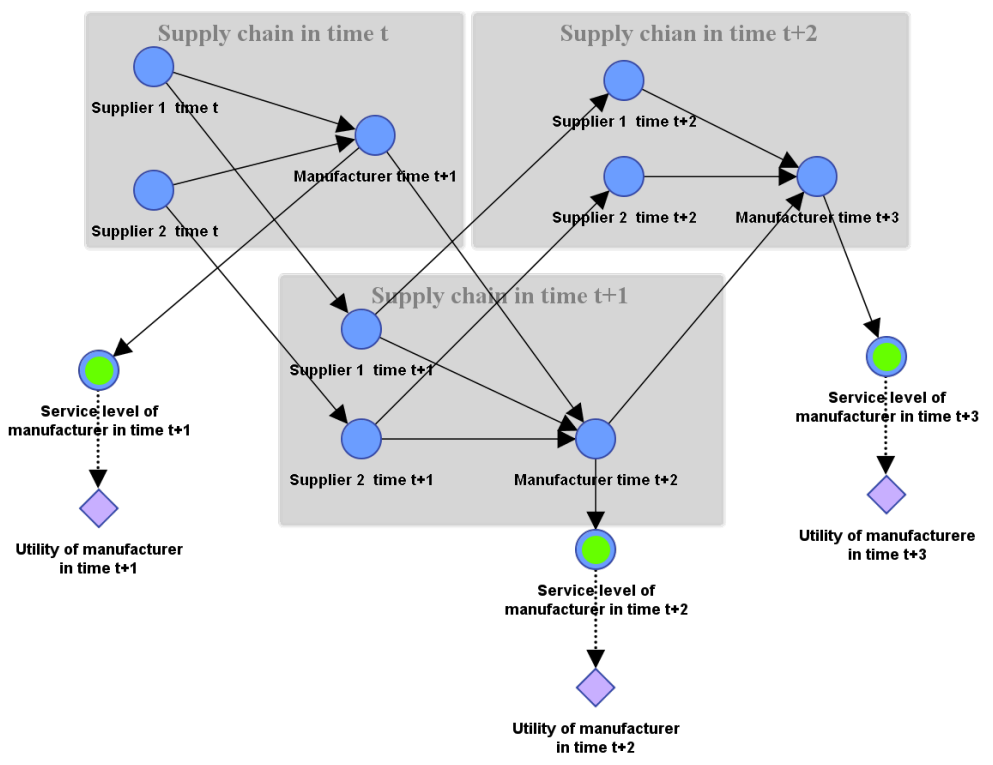

Figure 15: The DBN of two-stage supply chain with service level and utility of manufacturer consideration.

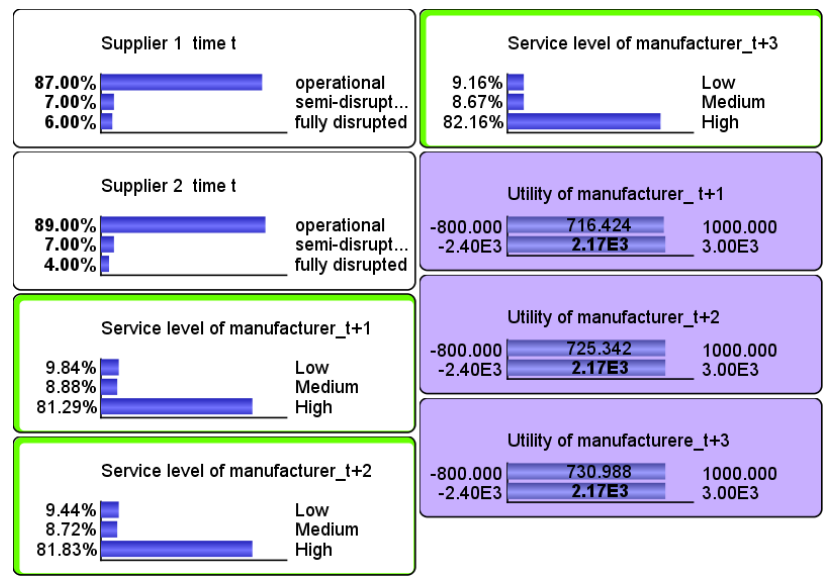

Figure 16: Distribution probability of SL and utility of manufacturer. 


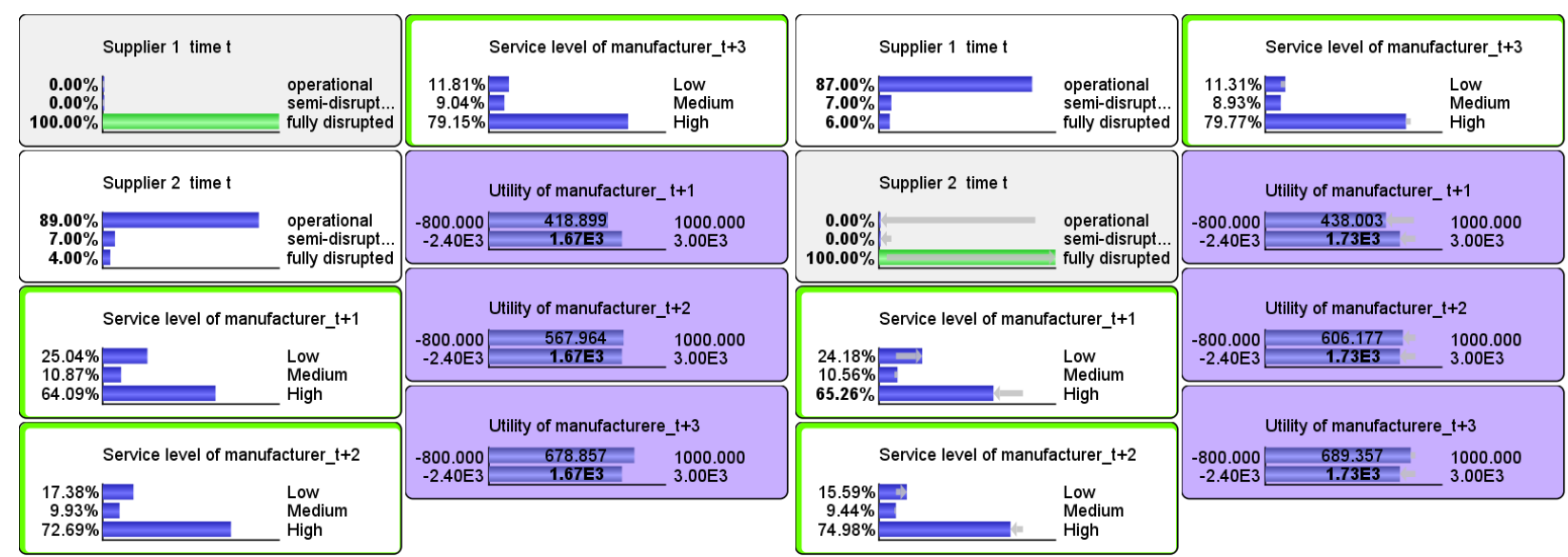

(a) Ripple effect of supplier 1 disruption on service level and util- (b) Ripple effect of supplier 1 disruption on service level and utility of manufacturer over three time periods.

ity of manufacturer over three time periods.

Figure 17: Ripple effect modeling of disruption of supplier 1 and 2 separately.

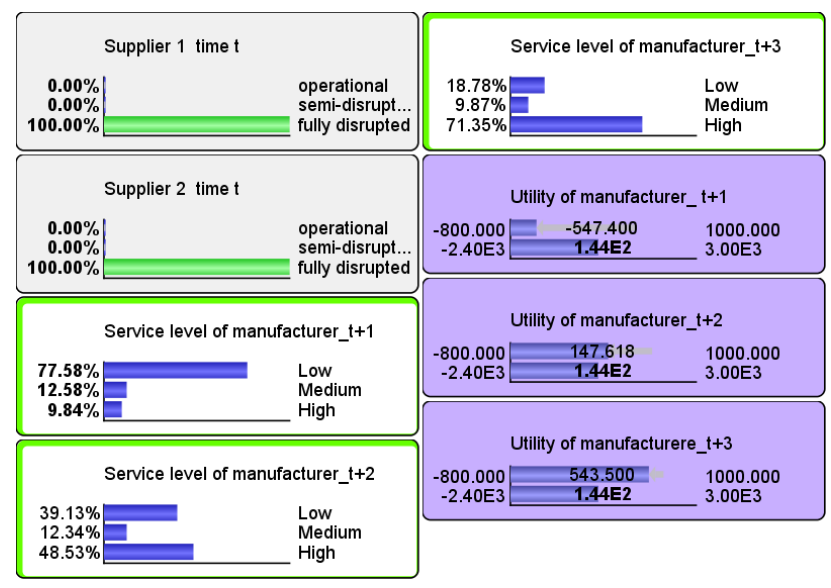

Figure 18: Ripple effect modeling of disruption of supplier 1 and 2 simultaneously.

The analysis of ripple effect study could assist SC management to identify critical suppliers whose disruption have higher impact on manufacturer or downstream entities of SC. Identifying and ranking vulnerable suppliers, particularly in a large supply network assist SC management to achieve a better risk mitigation decisions.

\section{Conclusion}

This study developed an integrated approach based on combining DTMC and DBN to model the ripple effect of a supplier disruption through the SC. We first proposed a discrete time Markov model with three states of operational, semi-disrupted and fully disrupted, in which these states are expressed in terms of the suppliers capacity. In order to be able to simulate and analyse the ripple effect in the supply chains, we equalised the Markov chain model with DBN.

Two substantive contributions emerge. First, our approach allows modelling the disruption propagation with consideration of changes in operational states at SC nodes. Notably, ripple effect assessment in multi-stage SCs is particularly challenged by consideration of both vulnerability and recoverability capabilities at individual firms in the network. We construct a new model based on integrating DTMC and DBN to quantify the ripple effect. We use DTMC to model the recovery and vulnerability of suppliers. The proposed DTMC model is then equalised with a 
DBN model in order to simulate the propagation behavior of supplier disruption in the SC. Second, we proposed a metric that quantifies the ripple effect of supplier disruption on manufacturer in terms of total expected utility and service level. The ripple effect measure constructed is examined and tested using two case-studies. The findings suggest that our model can be of value in revealing latent high-risk paths in the SC, analysing the performance impact of both a disruption and its propagation, and prioritizing the contingency and recovery policies.

Disruptions in SC are stochastic and dynamic in nature, meaning that there is a probability associated with the occurrence of disruption and the impact of disruption can propagate from time to time. DBN is a powerful tool for handling uncertainty and decision making in a dynamic environment. The DBN is utilized to model the ripple effect of supplier disruption on manufacturer over a different time horizon. We proposed metrics that could measure the ripple effect of any SC entities (e.g., supplier, manufacturer) in term of service level and expected utility with the help of inference analysis (evidential reasoning). The simulation results carried out in this study show how disruption of suppliers in the initial time period could propagate through the SC and negatively impact the service level of the manufacturer. The findings can help researchers and practitioners to identify vulnerable suppliers and simulate the propagation impact of their disruption on other entities of SC, particularly the ones located on the downstream.

There are several limitations and future research directions associated with this study. We developed metrics to measure the ripple effect of supplier disruption on manufacture in terms of TEU and service level dynamically. Ripple effect can be also measured in term of other SC performances such as lead time and market share loss [1,2]. The future research efforts can focus on developing metrics that include the above-mentioned performances in addition to TEU and service level. In this study, we consider a two-stage SC, however, future study should explore the disruption propagation impact of suppliers on distribution centers and retailers. The DBN is a powerful tool for simulating the disruption propagation of upstream entities on the downstream of the SC. Our integrated DTMC-DBN approach accounts for the ripple effect analysis in terms of the structural dynamics. Left ignored, however, remain the operational production-ordering decisions which in some cases may have a significant impact on the ripple effect [3, 23]. Finally, the probability elicitation of CPT could be exhaustive when the number of nodes in the DBN model increases exponentially. Hence, alternative methods such as Noisy - OR method can be used to reduce the computational complexity of DBN with a large number of nodes.

\section{References}

[1] D. Ivanov, "Simulation-based ripple effect modelling in the supply chain," International Journal of Production Research, vol. 55, no. 7, pp. 2083-2101, 2017.

[2] D. Ivanov, B. Sokolov, and A. Dolgui, "The ripple effect in supply chains: trade-off efficiency-flexibility-resilience in disruption management," International Journal of Production Research, vol. 52, no. 7, pp. 2154-2172, 2014.

[3] A.Dolgui, D.Ivanov, and M.Rozhkov, "Does the ripple effect influence the bullwhip effect? an integrated analysis of structural and operational dynamics in the supply chain.." in review.

[4] EETimes, IBM ships processors for Nintendo's Wii. http://www.eetimes.com/document.asp?.id=1163426.

[5] J. Blackhurst, M. J. Rungtusanatham, K. Scheibe, and S. Ambulkar, "Supply chain vulnerability assessment: A network based visualization and clustering analysis approach," Journal of Purchasing and Supply Management, vol. 24, no. 1, pp. 21 - $30,2018$.

[6] D. Ivanov, Structural Dynamics and Resilience in Supply Chain Risk Management. 112017.

[7] N. Bugert and R. Lasch, "Effectiveness of responsive pricing in the face of supply chain disruptions," Computers $\mathcal{E}$ Industrial Engineering, vol. 124, pp. $304-315,2018$.

[8] T. Sawik, "Disruption mitigation and recovery in supply chains using portfolio approach," Omega, vol. 84, pp. 232 - 248, 2019.

[9] B. Behdani, Z. Lukszo, and R. Srinivasan, "Agent-oriented simulation framework for handling disruptions in chemical supply chains," Computers $\mathcal{E}$ Chemical Engineering, 2018.

[10] H. Wang, T. Gu, M. Jin, R. Zhao, and G. Wang, "The complexity measurement and evolution analysis of supply chain network under disruption risks," Chaos, Solitons E Fractals, vol. 116, pp. 72 - 78, 2018.

[11] J. W. Wang, W. H. Ip, R. R. Muddada, J. L. Huang, and W. J. Zhang, "On petri net implementation of proactive resilient holistic supply chain networks," The International Journal of Advanced Manufacturing Technology, vol. 69, pp. 427-437, Oct 2013.

[12] D. Ivanov, R. Hartl, A. Dolgui, A. Pavlov, and B. Sokolov, "Integration of aggregate distribution and dynamic transportation planning in a supply chain with capacity disruptions and the ripple effect consideration," International Journal of Production Research, vol. 53, no. 23, pp. 6963-6979, 2015.

[13] D. Ivanov, B. Sokolov, I. Solovyeva, A. Dolgui, and F. Jie, "Dynamic recovery policies for time-critical supply chains under conditions of ripple effect," International Journal of Production Research, vol. 54, no. 23, pp. 7245-7258, 2016.

[14] D. Ivanov, A. Dolgui, B. Sokolov, and M. Ivanova, "Literature review on disruption recovery in the supply chain," International Journal of Production Research, vol. 55, no. 20, pp. 6158-6174, 2017.

[15] B. Sokolov, D. Ivanov, A. Dolgui, and A. Pavlov, "Structural quantification of the ripple effect in the supply chain," International Journal of Production Research, vol. 54, no. 1, pp. 152-169, 2016. 
[16] E. Levner and A. Ptuskin, "Entropy-based model for the ripple effect: managing environmental risks in supply chains," International Journal of Production Research, vol. 56, no. 7, pp. 2539-2551, 2018.

[17] R. Ojha, A. Ghadge, M. K. Tiwari, and U. S. Bititci, "Bayesian network modelling for supply chain risk propagation," International Journal of Production Research, vol. 0, no. 0, pp. 1-25, 2018.

[18] D. Ivanov, A. Dolgui, and B. Sokolov, "The impact of digital technology and industry 4.0 on the ripple effect and supply chain risk analytics," International Journal of Production Research, vol. 0, no. 0, pp. 1-18, 2018.

[19] K. P. Scheibe and J. Blackhurst, "Supply chain disruption propagation: a systemic risk and normal accident theory perspective," International Journal of Production Research, vol. 56, no. 1-2, pp. 43-59, 2018.

[20] J. Han and K. Shin, "Evaluation mechanism for structural robustness of supply chain considering disruption propagation," International Journal of Production Research, vol. 54, no. 1, pp. 135-151, 2016.

[21] C. S. Tan, P. S. Tan, and S. S. G. Lee, "Quantifying disruptions propagation in a supply chain," in 2015 IEEE International Conference on Industrial Engineering and Engineering Management (IEEM), pp. 1668-1672, Dec 2015.

[22] C. Otto, S. Willner, L. Wenz, K. Frieler, and A. Levermann, "Modeling loss-propagation in the global supply network: The dynamic agentbased model acclimate," Journal of Economic Dynamics and Control, vol. 83, pp. 232 - 269, 2017.

[23] D. Ivanov, "Disruption tails and revival policies: A simulation analysis of supply chain design and production-ordering systems in the recovery and post-disruption periods," Computers $\mathcal{E}$ Industrial Engineering, 2018.

[24] D. Ivanov, A. Pavlov, D. Pavlov, and B. Sokolov, "Minimization of disruption-related return flows in the supply chain," International Journal of Production Economics, vol. 183, pp. 503 - 513, 2017. Closed Loop Supply Chain (CLSC): Economics, Modelling, Management and Control.

[25] D. Ivanov, A. Pavlov, A. Dolgui, D. Pavlov, and B. Sokolov, "Disruption-driven supply chain (re)-planning and performance impact assessment with consideration of pro-active and recovery policies," Transportation Research Part E: Logistics and Transportation Review, vol. 90, pp. 7 - 24, 2016. Risk Management of Logistics Systems.

[26] D. Ivanov, A. Pavlov, and B. Sokolov, "Optimal distribution (re)planning in a centralized multi-stage supply network under conditions of the ripple effect and structure dynamics," European Journal of Operational Research, vol. 237, no. 2, pp. 758 - 770, 2014.

[27] D. Ivanov, A. Dolgui, and B. Sokolov, "Supply chain design with disruption considerations: Review of research streams on the ripple effect in the supply chain," IFAC-PapersOnLine, vol. 48, no. 3, pp. 1700 - 1707, 2015. 15th IFAC Symposium onInformation Control Problems inManufacturing.

[28] S. Hosseini and K. Barker, "A Bayesian network model for resilience-based supplier selection," International Journal of Production Economics, vol. 180 , pp. $68-87,2016$.

[29] S. Hosseini and K. Barker, "Modeling infrastructure resilience using bayesian networks: A case study of inland waterway ports," Computers $\mathcal{F}$ Industrial Engineering, vol. 93, pp. 252 - 266, 2016.

[30] S. Hosseini, A. A. Khaled, and M. Sarder, "A general framework for assessing system resilience using bayesian networks: A case study of sulfuric acid manufacturer," Journal of Manufacturing Systems, vol. 41, pp. 211 - 227, 2016.

[31] A. Qazi, A. Dickson, J. Quigley, and B. Gaudenzi, "Supply chain risk network management: A bayesian belief network and expected utility based approach for managing supply chain risks," International Journal of Production Economics, vol. 196, pp. 24 - 42, 2018.

[32] B. Song, C. Lee, and Y. Park, "Assessing the risks of service failures based on ripple effects: A bayesian network approach," International Journal of Production Economics, vol. 141, no. 2, pp. 493 - 504, 2013. Special Issue on Service Science.

[33] S. Hosseini, K. Barker, and J. E. Ramirez-Marquez, "A review of definitions and measures of system resilience," Reliability Engineering E System Safety, vol. 145, pp. 47 - 61, 2016.

[34] N. Fenton, Risk assessment and decision analysis with Bayesian networks. Boca Raton, FL: CRC Press, 2013

[35] B. Cai, Y. Liu, and Q. Fan, "A multiphase dynamic bayesian networks methodology for the determination of safety integrity levels," Reliability Engineering $\mathcal{E}$ System Safety, vol. 150, pp. 105 - 115, 2016.

[36] S. Rebello, H. Yu, and L. Ma, "An integrated approach for system functional reliability assessment using dynamic bayesian network and hidden markov model," Reliability Engineering $\mathcal{E}$ System Safety, vol. 180, pp. 124 - 135, 2018.

[37] S. Barua, X. Gao, H. Pasman, and M. S. Mannan, "Bayesian network based dynamic operational risk assessment," Journal of Loss Prevention in the Process Industries, vol. 41, pp. 399 - 410, 2016.

[38] B. G. Marcot and T. D. Penman, "Advances in bayesian network modelling: Integration of modelling technologies," Environmental Modelling $\mathcal{E}$ Software, 2018.

[39] S. Hosseini and M. Sarder, "Development of a bayesian network model for optimal site selection of electric vehicle charging station," International Journal of Electrical Power E Energy Systems, vol. 105, pp. 110 - 122, 2019.

[40] A. M. Ross, Y. Rong, and L. V. Snyder, "Supply disruptions with time-dependent parameters," Computers E Operations Research, vol. 35, no. 11, pp. 3504 - 3529, 2008. Part Special Issue: Topics in Real-time Supply Chain Management.

[41] L. Zheng, O. Mengshoel, and J. Chong, "Belief propagation by message passing in junction trees: Computing each message faster using gpu parallelization," in Proceedings of the Twenty-Seventh Conference on Uncertainty in Artificial Intelligence, UAI'11, (Arlington, Virginia, United States), pp. 822-830, AUAI Press, 2011. 